\title{
Keeping up with the nanopores
}

\section{By Tracey Baas, Senior Editor}

Speed and cost are the selling points for nanopore sequencing, which holds the promise of being faster than sequencing technologies currently on the market without needing extensive DNA sample preparation. But thus far speed has come at the cost of accuracy. Now, a team at the University of California, Santa Cruz has discovered a way to improve the accuracy of nanopore sequencing while retaining its speed. ${ }^{1}$

Oxford Nanopore Technologies Ltd. has licensed the technology and is incorporating it into its nanopore sequencing platforms.

The underlying premise of nanopore sequencing involves pushing a strand of DNA through a pore in a protein or silicon sheet. Because the pore is so small, a coiled nucleic acid molecule will unravel and progress single file through the pore. Each nucleotide produces a distinct signature as it passes through the pore, thus allowing for the sequencing of the nucleic acid chain.

At least five companies are developing nanopore-based devices that can sequence DNA. The companies have not divulged many details about their respective technologies, including the specific pores and what type of surface the pores are embedded in. The companies do have different strategies for pushing the DNA through the pore and for measuring the
DNA once it is inside the pore (see Table 1, "Nanopore sequencing in development").

Thus far, a key sticking point has been driving DNA through at a controlled speed that allows for accurate detection of each individual nucleotide's signature. Without any speed controls, DNA will shoot through a nanopore so rapidly that the number of nucleotides cannot be counted. ${ }^{2}$

To address the problem, the UCSC team combined phi29 DNA polymerase (DNAP) and blocking oligomers to move DNA through a nanopore at a more controlled speed than that which results from relying on just electrophoretic motion induced by an externally applied voltage.

The group previously showed that phi29 DNAP drives DNA strands through a nanopore as the polymerase replicates the DNA. The force of the voltage-induced electric field, acting in the opposite direction of the replication, helps hold the DNA strand taut and reduces its speed through the nanopores. ${ }^{3}$ The problem was that replication occurred not just at the nanopore, but also in solution, depleting reagents before they reached the nanopore for detection.

To get around that issue, the team turned to blocking oligomers, which protect DNA from polymerase-mediated replication until the DNA-DNAPblocking oligomer complex reaches the nanopore.

Specifically, the DNA is pulled through the nanopore in one direction by an applied voltage, which leads to unzipping of the blocking oligomer selectively at the pore. The DNA is then driven in the opposite direction through the nanopore by DNAP-mediated DNA replication. The forward and reverse motion of the DNA through the pore allows for two readouts per nucleotide.

As proof of concept, the group used $\alpha$-hemolysin nanopores embedded in a lipid bilayer and processed about 500 DNA molecules at 130 molecules per hour per pore. They used an ionic current map to count the number of nucleotides that passed through the pore, but they did not identify the bases.

Table 1. Nanopore sequencing in development. At least five companies are developing nanopore-based DNA sequencers, which determine base-pair identity by measuring unique signatures generated by each nucleotide as it passes through a pore. Three key design choices distinguish nanopore-based sequencing systems in development: the composition of the nanopore itself, which could be a solid-state material or a biological material consisting of a recombinant channel protein such as a porin; the DNA transport method, which controls how fast nucleotides pass through the pore and is critical to ensure accurate sequencing; and the nature of the signature generated by the DNA as it passes through the pore.

\begin{tabular}{lllll}
\hline Company & $\begin{array}{l}\text { Nanopore } \\
\text { composition }\end{array}$ & DNA transport method & $\begin{array}{l}\text { Detection } \\
\text { signature }\end{array}$ & $\begin{array}{l}\text { Predicted } \\
\text { commercial launch }\end{array}$ \\
\hline Genia Technologies Inc. & Biologic & $\begin{array}{l}\text { Electronic control moves DNA through nanopores embedded in a } \\
\text { lipid bilayer }\end{array}$ & Ionic current & 2013 \\
\hline
\end{tabular}

IBM Research/Arizona

Technology Enterprises/

Roche (SIX:ROG;

Solid state

Electronic control moves DNA through nanopores embedded in a Electron tunneling silicon chip current

Undisclosed

OTCQX:RHHBY)/454 Life

Sciences Corp.

NABsys Inc.

Solid state

Electronic control moves DNA with hybridized oligonucleotide probes through nanopores embedded in a silicon chip $^{A}$

Ionic current

Undisclosed

\begin{tabular}{|c|c|c|c|c|}
\hline NobleGen Biosciences Inc. & Solid state & $\begin{array}{l}\text { Electronic control moves synthetic DNA template with hybridized } \\
\text { oligonucleotide beacons through nanopores in a silicon chip, } \\
\text { displacing the beacons }{ }^{B}\end{array}$ & Fluorescence & 2014 \\
\hline $\begin{array}{l}\text { Oxford Nanopore } \\
\text { Technologies Ltd. }\end{array}$ & Biologic & $\begin{array}{l}\text { Processive enzyme moves DNA through nanopores embedded in } \\
\text { a lipid bilayer }\end{array}$ & Ionic current & $2 \mathrm{H} 12$ \\
\hline
\end{tabular}


The researchers opted not to sequence the DNA-instead measuring the accuracy of their approach by counting nucleotides-because the prototype nanopore used, $\alpha$-hemolysin, does not provide sufficient resolution to read bases. The estimated rate of DNA template error was about $10 \%-25 \%$.

Results were published in Nature Biotechnology.

"It is nice work demonstrating enzymatic motion control of DNA, but it also shows that the field is a long way from having a commercial nanopore sequencing machine," said Jonathan Rothberg, founder, chairman and CEO of Ion Torrent Systems Inc. "I would not at this time say they are sequencing since they did not determine the sequence. Thus far they have only been able to compare signals to what would be expected for the known sequence they used."

Nevertheless, said Rothberg,

"It is nice work

demonstrating enzymatic motion control of DNA, but it also shows that the field is a long way from having a commercial nanopore sequencing machine."

- Jonathan Rothberg, Ion Torrent Systems Inc. the paper "is the most important recent progress in the field. It allows DNA to go through the pore in a way that allows the sequence to be seen."

Ion Torrent is not using nanopore sequencing and instead is developing its benchtop Ion Personal Genome Machine Sequencer and the benchtop Ion Proton Sequencer, which use polymerase-driven DNA replication combined with a proton-based readout to enable the $\$ 1,000$ genome. Commercial launch is mid-2012.

The paper "convincingly demonstrates that one of the major challenges of nanopore-based sequencing-movement control of DNA through the nanopore-can be overcome using an enzymatic approach," said Stefan Roever, CEO of Genia Technologies Inc. "The ability to read the same molecule multiple times, possibly forward and reverse, as demonstrated in the work, is also a significant factor in reducing error rates."

Genia is developing its Last Gen sequencing platform, which uses biological nanopores embedded in a lipid bilayer with a readout based on ionic current disruption. The company expects commercial availability in 2013.

"I think the work is a nice experimental method showing that DNA motion can be slowed down, but I don't think the actual experimental design will ever be used in practice for sequencing," said Charles Cantor, CSO for Sequenom Inc. "The blocking polymers that they used are complementary to the DNA template that they sequenced. This would require one to know the sequence a priori to sequencing. Perhaps, as a long shot, the method could be extended to using a panel of generic blocking oligomers, but that would require a whole different set of proofof-concept experiments."
"They also use a rather simplistic DNA template so that they avoid problems with secondary structure. I would be interested to see if they could reproduce their findings using longer, more typical DNA sequences," he told SciBX.

The Sequenom Center for Molecular Medicine Inc., a subsidiary of Sequenom Inc., is developing a broad range of diagnostics with a focus on prenatal diseases and conditions, marketed under the name SensiGene.

\section{Implementing the nanopores}

Roever did say the paper's current template register error rate is unusable for a commercial sequencing technique. He thinks accuracy could be improved by including a "readout of an additional signature of any enzymatic activity-essentially an electric signal indicating the strand has been advanced a single frame-or by reading the signal from several bases in the pore at a time" and then using a deconvolving algorithm to link a specific base with its specific signal.

"Ideally, a combination of both techniques would be used," he added.

Mark Akeson, chair of biomolecular engineering at UCSC and lead author on the Nature Biotechnology paper, told SciBX, "The error rate has already been substantially improved and systematized for a working commercial instrument by Oxford Nanopore," which holds rights to patents covering the technology described in the paper.

An Oxford Nanopore spokesperson said, "We are constantly iterating the system to improve error rates, and we are targeting between $0.1 \%-2 \%$ for our launch product," adding that the company's chemistry "is not the published chemistry of our academic partners. Our enzyme and nanopore are undisclosed."

Regardless of approach, Ion Current's Rothberg thinks the next big issue for nanopore sequencing companies is fabrication.

"Nanopores, while being miniaturized, have no existing method to be easily fabricated," he said. "They will be difficult to manufacture and will have significant issues scaling to a practical parallel implementation."

Baas, T. SciBX 5(11); doi:10.1038/scibx.2012.271

Published online March 15, 2012

\section{REFERENCES}

1. Cherf, G.M. et al. Nat. Biotechnol.; published online Feb. 14, 2012; doi:10.1038/nbt.2147

Contact: Mark Akeson, University of California, Santa Cruz, Calif. e-mail: makeson@soe.ucsc.edu

2. Branton, D. et al. Nat. Biotechnol. 26, 1146-1153 (2008)

3. Lieberman, K.R. et al. J. Am. Chem. Soc. 132, 17961-17972 (2010)

COMPANIES AND INSTITUTIONS MENTIONED

Genia Technologies Inc., Mountain View, Calif.

Ion Torrent Systems Inc., San Francisco, Calif.

Oxford Nanopore Technologies Ltd., Oxford, U.K.

Sequenom Center for Molecular Medicine Inc., Grand Rapids, Mich.

Sequenom Inc. (NASDAQ:SQNM), San Diego, Calif.

University of California, Santa Cruz, Calif. 